\title{
Nuclear EGFR in ductal invasive breast cancer: correlation with cyclin-D1 and prognosis
}

\author{
Ita Hadžisejdić ${ }^{1}$, Elvira Mustać ${ }^{1}$, Nives Jonjić ${ }^{1}$, Marija Petković ${ }^{2}$ and Blaženka Grahovac ${ }^{1}$ \\ ${ }^{1}$ Department of Pathology, School of Medicine, University of Rijeka, Rijeka, Croatia and ${ }^{2}$ Department of \\ Oncology, Clinical Hospital Centre Rijeka, University of Rijeka, Rijeka, Croatia
}

\begin{abstract}
The epidermal growth factor receptor (EGFR)-family and cyclin-D1 have been extensively studied in breast cancer; however systematic studies that examine protein expression and gene status in the same cohort of patients are lacking. Also emerging evidences suggest existence of a direct EGFR-signaling pathway, which involves cellular transport of EGFR from cell membrane to the nucleus, and transcriptional regulation of the target genes. Thus, we examined the protein expression of membrane EGFR, nuclear EGFR, cyclin-D1 and the corresponding gene status in $\mathbf{1 1 3}$ breast carcinomas by immunohistochemistry and fluorescence in situ hybridization using tissue microarrays. Membrane EGFR overexpression and EGFR gene amplification were detected in $2 \%$ cases, while nuclear EGFR was detected in $40 \%$ of cases, with $12 \%$ having high nuclear EGFR staining. Nuclear EGFR correlated with tumor size $(P=0.0005)$, lymph node metastasis $(P=0.0288)$, Nottingham prognostic index $(P=0.0011)$ and estrogen receptor $(E R)$ expression $(P=0.0258)$ but the letter correlation was observed only in premenopausal group of patients. Strong cyclin-D1 expression and cyclin-D1 gene (CCND1) amplification were found in 64 and $13 \%$ of the cases, respectively. Cyclin-D1 expression showed positive correlation with ER $(P=0.0113)$ and inverse correlation with Nottingham prognostic index $(P=0.0309)$ and membrane EGFR $(P=0.0201)$. CCND1 amplification also showed inverse correlation with membrane EGFR $(P=0.0420)$. A strong correlation between membrane EGFR expression and gene amplification $(P=0.0035)$, as well as cyclin-D1 overexpression and gene amplification $(P=0.0362)$, was demonstrated. On univariate analysis cyclin-D1 expression showed a correlation with longer overall survival in the premenopausal group and nuclear EGFR correlated with shorter overall survival in whole cohort as well in the premenopausal group of patients. Multivariate analysis revealed nuclear EGFR to be an independent prognostic factor and showed 3.4 times greater mortality risk for nuclear EGFR +++ patients as compared with nuclear EGFR negative patients (hazard ratio $=3.402 ; P=0.0026$ ).
\end{abstract}

Modern Pathology (2010) 23, 392-403; doi:10.1038/modpathol.2009.166; published online 8 January 2010

Keywords: breast cancer; EGFR family; nuclear EGFR; cyclin-D1; CCND1

Breast cancer is the most common type of cancer among women in the world, with incidence of more than a million and mortality of more than 500000 deaths annually. ${ }^{1}$ Although incidence rate is increasing globally, mortality rates are decreasing and this is mostly due to wide use of mammography as screening method, better treatment in early stages of the disease and new anticancer drugs. ${ }^{2}$ Advances

Correspondence: Professor B Grahovac, PhD, Department of Pathology, School of Medicine, University of Rijeka, Braće Branchetta 20, Rijeka 51 000, Croatia.

E-mail: blazenka.grahovac@medri.hr

Received 19 July 2009; revised 19 August 2009; accepted 25 September 2009; published online 8 January 2010 in molecular medicine have facilitated identification of tumor markers that not only predict prognosis and therapeutic response, but also are target of novel anticancer drugs. Breast cancer is frequently defined as a heterogeneous disease because there is a percentage of patients who behave in an unexpected way even if clinical and pathological parameters indicate the opposite. ${ }^{3}$ Although parameters such as tumor size, lymph node status, histological grade and proliferative index are essential, they are still not enough if we want to provide the best therapeutic option and consequently the best prognosis to the patients. HER-2, epidermal growth factor receptor (EGFR) and cyclin-D1 are proteins associated with tumorigenesis and prognostics 
factors of breast cancer. ${ }^{4}$ Development of novel drugs specifically targeted to these molecules has attracted considerable interest in evaluating the overall status and relationships between these proteins. HER-2 is overexpressed in 15-30\% of invasive breast cancers, and it has been associated with poor prognosis with shorter disease-free and overall survival; moreover, it has been a predictive factor for response to chemotherapy, antiestrogens, and herceptin. ${ }^{5,6}$ Although, in breast cancer, EGFR is reported to be expressed in 5-91\%, ${ }^{4,7,8}$ and in several studies is also associated with poor prognosis, ${ }^{9,10}$ there is still no clear evidence regarding its prognostic relevance. On the other hand, emerging evidence suggest the existence of a new mode of EGFR-signaling pathway in which activated EGFR undergoes nuclear translocation and subsequently regulates gene expression. ${ }^{11}$ Nuclear EGFR functions as a transcription cofactor that interacts with and activates the $C C N D 1$ gene, a key positive regulator of proliferation. ${ }^{12}$ Cyclin-D1 protein plays an important role in the regulation of the $G_{1}$ phase of the cellcycle where it binds to cyclin-dependent kinase-4 (cdk4) or cdk6 to promote the phosphorylation of retinoblastoma protein $(\mathrm{pRb})$. Overexpression is reported to be more prevalent than amplification (amplification ranges 9-24\%), with reported frequency ranging from 28 to $83 \% .^{13}$ Cyclin-D1 overexpression with or without CCND1 amplification has received great attention in the literature in the past few years due to results of in vitro studies and data from clinical trials implicating cyclin-D1 overexpression in resistance to tamoxifen treatment. ${ }^{14-16}$ Transcription of cyclin-D1 is regulated through the estrogen receptor (ER) and also it has been suggested that cyclin-D1 can directly bind to and activate ER independently from estrogen. ${ }^{16,17}$ Cyclin-D1 is also a downstream target of HER-2 and EGFR. ${ }^{18}$ It is evident that there is a complex interplay between these proteins whose clinical implications nowadays are brought even more under the spotlight knowing the fact that specific drugs aiming at these molecules are readily available.

The prognostic significance of nuclear EGFR has recently been demonstrated in the study of Lo et $a ;^{19}$ however, the clinical implications of nuclear EGFR in the context of other histopathological parameters have not been evaluated. Thus, the aims of this study are to analyze the expression of nuclear EGFR and cyclin-D1, as well as HER-2 and membrane EGFR, and the corresponding gene status in invasive ductal carcinoma and correlate it with other clinicopathological features and overall survival.

\section{Materials and methods}

\section{Patients and Tumor Specimens}

The tissue microarrays were constructed and they contained replicate $1-\mathrm{mm}$ cores of 117 invasive ductal breast carcinoma. During immunohistochemical procedure and in situ hybridization some cores were either lost, fragmented, did not have invasive tumor or showed suboptimal hybridization signals; thus the number of examined tumors differed between analyses. All patients included in the study were newly diagnosed with breast cancer at the Department of Pathology, School of Medicine, University of Rijeka, from 2000 to 2002. Criteria for inclusion were: histopathological diagnosis of invasive ductal carcinoma NOS type, availability of paraffin-embedded tissue specimens and clinical data. All carcinomas were classified according to the criteria of the WHO. Histological grade was determined according to the modified BloomRichardson classification ${ }^{20}$ and size was categorized according to the TNM staging criteria. ${ }^{21}$ The Nottingham prognostic index was calculated as previously described. ${ }^{22}$ Clinicopathological information, including patient age at initial diagnosis, tumor size, lymph node metastasis, estrogen (ER) and progesterone receptors (PR), lymphovascular invasion and menopause status were obtained from patient records. All patients had the same followup time of 60 months and survival duration was defined as the time from surgery to death attributed to breast cancer. After surgery (in every case where it was possible partial mastectomy was performed instead of total mastectomy), depending on the extent of disease, patients received radiation therapy, adjuvant chemotherapy (FEC: 5-fluorouracil, epirubicin, cyclophosphamide; FAC: 5-fluorouracil, doxorubicin, cyclophosphamide; CMF: cyclophosphamide, methotrexate, 5-fluorouracil or AC: adriamycin, cyclophosphamide protocol) and hormonepositive patients received tamoxifen ( \pm goserelin if premenopausal). Patients who were HER-2-positive received a 1-year trastuzumab regimen.

\section{Immunohistochemical Staining}

Tissue microarray slides were deparaffinized in xylene substitute, rehydrated through three changes of alcohol and subjected to antigen retrieval. Depending on the antibody used we used the appropriate antigen retrieval protocol according to manufacturer's instructions. For HER-2 (HercepTest; DakoCytomation, Glostrup, Denmark), cyclin-D1 (SP4, 1:100; Neomarkers, Fremont, USA) and Ki-67 (MIB-1, 1:75; Dako, Glostrup, Denmark) antigen retrieval was performed by microwave oven treatment of slides in $10 \mathrm{mM}$ sodium citrate ( $\mathrm{pH} 6.0$ ), while for membrane EGFR (E30; Dako, Glostrup, Denmark) proteinase-K was used. For nuclear EGFR (EGFR.25, ready to use diluted 1:25; Novocastra, Newcastle upon Tyne, UK) immunostaining we made some minor modifications to the protocol described previously. ${ }^{19}$ We used double-antigen retrieval treatment, boiling of slides in citrate buffer and incubation with $0.025 \%$ trypsin in PBS (pH 7.4) 
for $8 \mathrm{~min}$. Following antigen retrieval the subsequent steps were performed with an automatic immunostainer (Dako Autostainer Plus; DakoCytomation, Fort Collins, CO, SAD) according to manufacturer's instructions, using appropriate DakoREAL solutions (Dako, Glostrup, Denmark). Used membrane EGFR (Dako, Denmark) antibody is raised against the $\mathrm{NH}_{2}$ terminus and recognizes only membrane EGFR in paraffin-embedded tissue, while nuclear EGFR (Novocastra, UK) antibody is raised against the $\mathrm{COOH}$ terminus and recognizes both membrane and nuclear EGFR. ${ }^{19}$

\section{Immunohistochemical Scoring}

For HER-2 staining we used a scoring system according to ASCO/CAP 2007 guidelines. ${ }^{23}$ CyclinD1 immunostaining was evaluated by the Allred score method. ${ }^{13,21,24}$ With this method, the intensity of the immunohistochemical reaction was recorded as follows: 0, negative (no staining of any nuclei even at high magnification); 1 , weak (only visible at high magnification); 2, moderate (readily visible at low magnification) or 3, strong (strikingly positive even at low magnification). The proportion of tumor nuclei showing positive staining was also recorded as either 0 (no staining), 1 ( $<1 \%$ nuclear staining), 2 $(1-10 \%), 3(11-33 \%), 4(34-66 \%)$ and $5(67-100 \%$ nuclear staining). The proportion and intensity scores were subsequently added to obtain a total score, which ranged from 0 to 8 . Tumors were categorized into four groups: negative/weak (total scores 0-2), moderate (total scores 3-5) and strong (total scores 6-8) expression. Only nuclear staining was considered specific. Ki-67 was scored low if less than $10 \%$ of the nuclei of neoplastic cells were positive, intermediate if $10-30 \%$ of the nuclei of neoplastic cells were positive and high if more than $30 \%$ of the nuclei of neoplastic cells were positive. ${ }^{13}$ Membrane EGFR was assessed according to the following scoring approach: 0 (no immunoreactivity or immunoreactivity in $<10 \%$ of tumor cells); $1+$ (faint weak and incomplete staining in $>10 \%$ of tumor cells), $2+$ (weak-to-moderate complete membrane immunoreactivity in $>10 \%$ of tumor cells) and $3+$ (strong complete membrane immunoreactivity in $>10 \%$ of tumor cells). ${ }^{3}$ Nuclear EGFR was ranked into four groups according to the percentage of the positive tumor cells: high $(+++,>35 \%)$, medium $(++, 18-35 \%)$, low $(+$, $1-17 \%)$ and negative $(-, 0 \%){ }^{19}$

\section{Fluorescence In Situ Hybridization (FISH)}

For proteolytic pretreatment of tissue microarray slides, a commercial kit was used (Paraffin pretreatment reagent kit; Vysis, Downers Grove, IL, USA) according to the manufacturer's instructions. SpectrumOrange-labeled, gene-specific probes were used together with SpectrumGreen-labeled probes for the respective centromere region as a reference. All probes used were from Abbott (Vysis, Downers Grove, IL, USA). The probe combinations were HER2/centromere 17 (PathVysion), EGFR (LSI EGFR SpectrumOrange)/centromere 7 (CEP 7 SpectrumGreen) and CCND1 (LSI cyclin-D1 SpectrumOrange)/centromere 11 (CEP 11 SpectrumGreen). Briefly, tissue microarray sections were deparaffinized in xylene substitute, rinsed in $100 \%$ ethanol and air dried. Subsequently, slides were incubated in $0.2 \mathrm{~N} \mathrm{HCl}$ for $20 \mathrm{~min}$, rinsed in $2 \times \mathrm{SSC}(\mathrm{pH} 7.0)$ and immersed for $30 \mathrm{~min}$ in $1 \mathrm{M} \mathrm{NaSCN}$ solution pre-warmed at $80^{\circ} \mathrm{C}$. After proteinase digestion, slide denaturation $\left(95^{\circ} \mathrm{C}\right.$ for $\left.5 \mathrm{~min}\right)$ and hybridization $\left(37^{\circ} \mathrm{C}\right.$ overnight) were performed in HYBrite (Vysis, IL, USA). Following day, slides were washed, counterstained with DAPI (Vysis, Downers Grove, IL, USA) and examined with a fluorescence microscope (Olympus BX50, Tokyo, Japan).

\section{FISH Scoring}

For all genes evaluation of in situ hybridization was performed by counting gene-specific signals and the corresponding control signals in 40 nuclei, at two or three different areas of invasive component of neoplasia. When evaluating the HER-2 gene if the ratio between $H E R-2 / C E P 17$ signals was $>2.2$ the HER-2 gene was considered amplified and when the ratio was $<1.8$, the $H E R-2$ gene was considered not amplified. If the ratio was between 1.8 and 2.2 the result was categorized as equivocal. ${ }^{23}$ For EGFR and CCND1 a gene was considered amplified if the ratio of oncogene/cetromere was $\geq 2.0$.

\section{Statistical Analysis}

The PowerMarker 3.0 software package and $R$-statistical tools were used for all statistical calculations. ${ }^{25}$ Correlations between categorical variables were performed using Fisher's exact test and $\chi^{2}$-test. Overall survival was expressed as the number of months from diagnosis to the occurrence of an event (disease-related death). Cumulative survival probabilities were calculated by Kaplan-Meier method. The differences between survival rates were tested with log-rank test. All tests were two-tailed, with a 95\% confidence interval. Multivariate analysis was performed using the Cox multiple-hazards model. A $P$-value of 0.05 in the univariate survival analysis was adopted as the limit for inclusion in the multivariate model, and cases with missing values were excluded from this analysis.

\section{Results}

\section{Cyclin-D1 Expression in Invasive Ductal Carcinoma}

The correlations between cyclin-D1 expression, clinicopathological features, immunohistochemical 
Table 1 Correlations between cyclin-D1 expression, clinicopathological parameters and EGFR-family proteins in invasive ductal breast cancer

\begin{tabular}{|c|c|c|c|c|c|c|}
\hline Parameters & No. & $N A$ & $\begin{array}{c}\text { Cyclin-D1 } \\
\text { (negative/ } \\
\text { weak) }\end{array}$ & $\begin{array}{l}\text { Cyclin-D1 } \\
\text { (moderate) }\end{array}$ & $\begin{array}{l}\text { Cyclin-D1 } \\
\text { (strong) }\end{array}$ & $\begin{array}{c}\text { Fisher's } \\
\text { exact } \\
\text { test }\end{array}$ \\
\hline Overall & 113 & 4 & 10 & 31 & 72 & \\
\hline Tumor size & 113 & 4 & & & & 0.0691 \\
\hline $\mathrm{T} 1(\leqslant 2 \mathrm{~cm})$ & & & 5 & 11 & 46 & \\
\hline $\mathrm{T} 2(>2-5 \mathrm{~cm})$ & & & 5 & 18 & 22 & \\
\hline T3 $(>5 \mathrm{~cm})$ & & & 0 & 2 & 4 & \\
\hline Grade & 113 & 4 & & & & 0.4803 \\
\hline I & & & 3 & 6 & 22 & \\
\hline II & & & 4 & 16 & 38 & \\
\hline III & & & 3 & 9 & 12 & \\
\hline$L N$ mets & 113 & 4 & & & & 0.5395 \\
\hline 0 & & & 5 & 14 & 42 & \\
\hline $1-3$ & & & 2 & 5 & 14 & \\
\hline$\geqslant 4$ & & & 3 & 12 & 16 & \\
\hline$E R$ & 113 & 4 & & & & 0.0113 \\
\hline - & & & 5 & 19 & 22 & \\
\hline+ & & & 5 & 12 & 50 & \\
\hline$P R$ & 113 & 4 & & & & 0.104 \\
\hline- & & & 6 & 14 & 22 & \\
\hline+ & & & 4 & 17 & 50 & \\
\hline NPI & 112 & 5 & & & & 0.0309 \\
\hline Good $(<3.4)$ & & & 3 & 11 & 43 & \\
\hline $\begin{array}{l}\text { Moderate } \\
(3.4-5.4)\end{array}$ & & & 4 & 8 & 19 & \\
\hline Poor (>5.4) & & & 2 & 12 & 10 & \\
\hline$L V I$ & 79 & 38 & & & & 1 \\
\hline- & & & 1 & 5 & 15 & \\
\hline+ & & & 4 & 15 & 39 & \\
\hline Ki-67 & 113 & 4 & & & & 0.1681 \\
\hline$<10 \%$ & & & 3 & 16 & 30 & \\
\hline $10-30 \%$ & & & 2 & 10 & 30 & \\
\hline$>30 \%$ & & & 5 & 5 & 12 & \\
\hline Nuclear EGFR & 112 & 5 & & & & 0.5132 \\
\hline 0 & & & 8 & 17 & 42 & \\
\hline+ & & & 2 & 5 & 19 & \\
\hline++ & & & 0 & 3 & 4 & \\
\hline+++ & & & 0 & 6 & 7 & \\
\hline Membrane EGFR & 112 & 5 & & & & 0.0201 \\
\hline 0 & & & 6 & 28 & 66 & \\
\hline $1+$ & & & 4 & 3 & 3 & \\
\hline $2+$ & & & 0 & 0 & 1 & \\
\hline $3+$ & & & 0 & 0 & 1 & \\
\hline EGFR gene & 110 & 7 & & & & 0.2302 \\
\hline Not amplified & & & 8 & 30 & 70 & \\
\hline Amplified & & & 1 & 0 & 1 & \\
\hline HER-2 & 112 & 5 & & & & 0.5691 \\
\hline 0 & & & 7 & 19 & 44 & \\
\hline $1+$ & & & 0 & 4 & 13 & \\
\hline $2+$ & & & 2 & 2 & 6 & \\
\hline $3+$ & & & 1 & 6 & 8 & \\
\hline HER-2 gene & 113 & 4 & & & & 0.1826 \\
\hline Not amplified & & & 7 & 23 & 62 & \\
\hline Amplified & & & 3 & 8 & 10 & \\
\hline CCND1 & 112 & 5 & & & & 0.0362 \\
\hline Not amplified & & & 10 & 30 & 57 & \\
\hline Amplified & & & 0 & 1 & 14 & \\
\hline
\end{tabular}

CCND1, cyclin-D1 gene; EGFR, epidermal growth factor receptor; ER, estrogen receptor; Grade, histological grade; LN mets, lymph node metastasis; LVI, lymphovascular invasion; NA, not available; NPI, Nottingham prognostic index; PR, progesterone receptor.

and FISH findings are summarized in Table 1. In total, 113 tumor samples were examined and classified into four cyclin-D1 expression groups.
Six tumors (5\%) were classified as negative, $4(3 \%)$ as weak, $31(27 \%)$ as moderate and 72 $(64 \%)$ as strong-expressing samples, according to the Allred scoring method (Figure 1). Cyclin-D1 expression showed a statistically significant direct correlation with expression of ER $(P=0.0113)$, and inverse correlation with the Nottingham prognostic index $(P=0.0309)$ and expression of membrane EGFR $(P=0.0201)$. The same correlations of cyclin-D1 expression with clinicopathological features, immunohistochemical and FISH findings were found when tumors were grouped as post and premenopausal. Correlations with all other clincopathological features, immunohistological and FISH findings were not statistically significant (Table 1).

\section{CCND1 Status in Invasive Ductal Carcinoma}

The correlations between CCND1 amplification, clinicopathological features, immunohistochemical and FISH findings are summarized in Table 2. Out of the 112 tumor samples, 15 (13\%) showed amplification of the CCND1 gene and 97 (87\%) did not have amplification of the CCND1 gene (Figure 1). We found only statistically significant inverse correlation of CCND1 with expression of membrane EGFR $\left(P=0.0420, \chi^{2}\right.$-test $)$. No other correlations were found. The same correlations of CCND1 with clinicopathological features, immunohistochemical and FISH findings were found when tumors were grouped as post and premenopausal. Data on CCND1 amplification and overexpression were available for 112 cases. A statistically significant correlation between CCND1 amplification and cyclin-D1 overexpression was found (Table 1, $P=0.0362$ ). Out of 15 tumors with CCND1 amplification, 14 showed strong cyclin-D1 expression and one showed moderate expression (Table 1).

\section{Nuclear EGFR Expression in Invasive Ductal Carcinoma}

The correlations between nuclear EGFR expression, clinicopathological features, immunohistochemical and FISH findings are summarized in Table 3. In total 113 tumor samples were analyzed for nuclear EGFR and we detected in $13(12 \%)$ cases high $(+++)$ percentage of tumor cells to be positive for nuclear EGFR. Seven cases $(6 \%)$ had moderate $(++)$ percentage of tumor cells positive for nuclear EGFR, while $26(23 \%)$ had low $(+)$ percentage of tumor cells positive for nuclear EGFR. In the remaining $67(59 \%)$ cases we did not detect nuclear EGFR. Considering these data, nuclear staining for EGFR was detected in $40 \%$ of the tumor tissues we examined. Figure 1 shows representative tumors for 0/ + nuclear EGFR, +++ nuclear EGFR and negative nuclear EGFR, and +++ membrane EGFR. Both EGFR antibodies used successfully 

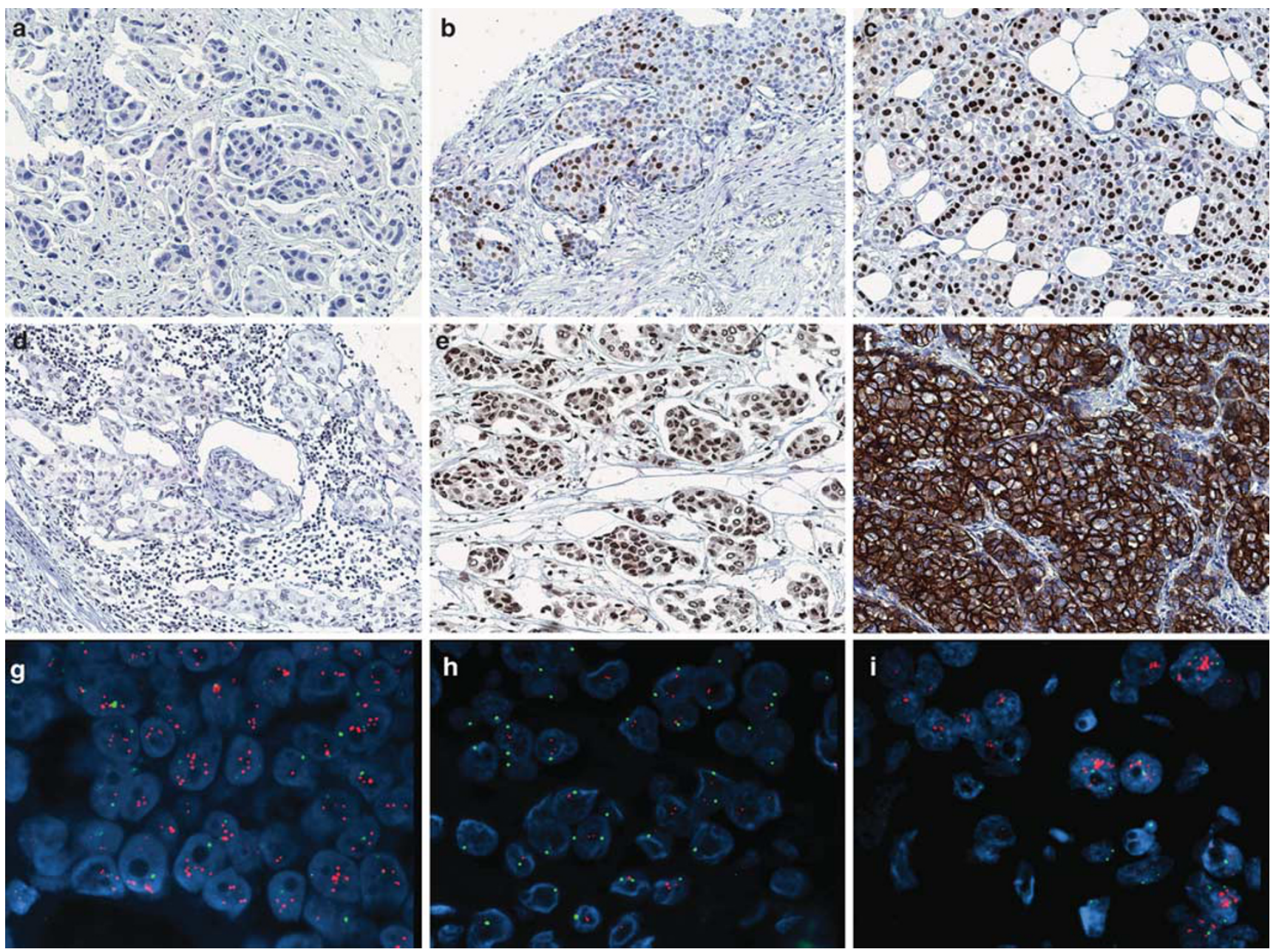

Figure 1 Grade-III invasive ductal carcinoma without cyclin-D1 expression (a) lacking CCND1-gene amplification (h). Grade-I invasive ductal carcinoma showing moderate cyclin-D1 expression (b). Grade-I invasive ductal carcinoma with strong cyclin-D1 expression (c) and CCND1-gene amplification (g). Invasive ductal carcinoma negative for nuclear EGFR (d) and tumor showing +++ nuclear EGFR staining (e). Invasive ductal breast cancer with $3+$ membrane EGFR staining and negative nuclear EGFR (f), with EGFR-gene amplification (i). For immunohistochemical images, $\times 200$ magnification; for FISH, $\times 1000$ magnification.

recognized membrane EGFR with 100\% concordance (data shown in the Supplementary information). In our sample only two tumors showed overexpression of membrane EGFR as stained with Dako's and Novocastra's antibody (data shown in the Supplementary information). Nuclear EGFR showed statistically significant correlation with tumor size $(P=0.0005)$, lymph node involvement $(P=0.0288)$ and Nottingham prognostic index $(P=0.0011)$. We did not find statistically significant correlation between nuclear EGFR and Ki-67 and cyclin-D1, both indicators of cell proliferation (Table 3). However, when tumors were grouped as post and premenopausal, we found additional statistically significant inverse correlation of nuclear EGFR with expression of ER $(P=0.0258$; data not shown), but just in the premenopausal group. HER-2 and membrane EGFR immunohistochemistry and FISH results are presented in the Supplementary information.

\section{Survival Analysis}

Univariate survival analysis of all tumor samples revealed tumor size, histological grade, lymph node metastasis, PR, Nottingham prognostic index and nuclear EGFR as prognostic factors for overall survival (Table 4 and Figure 2). Clinical significance of membrane EGFR overexpression and EGFR amplification could not be independently evaluated due to small number of informative cases, while HER-2 gene amplification showed borderline significance (Table 4). Tumors with strong cyclin-D1 expression showed better overall survival, but only in the premenopausal group of patients $(P=0.0033)$; however, there was no statistical significance in the postmenopausal group $(P=0.5365)$ or the whole patient cohort $(P=0.1538)$, although a trend toward better overall survival has been noticed (Table 4). We paired cyclin-D1 expression and CCND1 amplification with tumor size, histological grade, lymph node 
Table 2 Correlations between CCND1 amplification, clinicopathological parameters and EGFR-family proteins in invasive ductal breast cancer

\begin{tabular}{|c|c|c|c|c|c|}
\hline Parameters & No. & $N A$ & $\begin{array}{c}\text { CCND1 } \\
\text { not } \\
\text { amplified } \\
97\end{array}$ & $\begin{array}{c}\text { CCND1 } \\
\text { amplified } \\
15\end{array}$ & $\begin{array}{l}\text { Fisher's } \\
\text { exact test } \\
\text { (P-value) }\end{array}$ \\
\hline Tumor size & 112 & 5 & & & 0.210 \\
\hline $\mathrm{T} 1(\leqslant 2 \mathrm{~cm})$ & & & 52 & 9 & \\
\hline $\mathrm{T} 2(>2-5 \mathrm{~cm})$ & & & 41 & 4 & \\
\hline $\mathrm{T} 3(>5 \mathrm{~cm})$ & & & 4 & 2 & \\
\hline Grade & 112 & 5 & & & 0.380 \\
\hline I & & & 28 & 2 & \\
\hline II & & & 48 & 10 & \\
\hline III & & & 21 & 3 & \\
\hline$L N$ mets & 112 & 5 & & & 0.608 \\
\hline 0 & & & 52 & 8 & \\
\hline $1-3$ & & & 17 & 4 & \\
\hline$\geqslant 4$ & & & 28 & 3 & \\
\hline$E R$ & 112 & 5 & & & 0.094 \\
\hline- & & & 43 & 3 & \\
\hline+ & & & 54 & 12 & \\
\hline$P R$ & 112 & 5 & & & 0.783 \\
\hline- & & & 37 & 5 & \\
\hline+ & & & 60 & 10 & \\
\hline NPI & 111 & 6 & & & 0.818 \\
\hline Good $(<3.4)$ & & & 47 & 9 & \\
\hline Moderate (3.4-5.4) & & & 28 & 3 & \\
\hline Poor $(>5.4)$ & & & 21 & 3 & \\
\hline LVI & 79 & 38 & & & 0.499 \\
\hline- & & & 19 & 2 & \\
\hline+ & & & 48 & 10 & \\
\hline Ki-67 & 112 & 5 & & & 0.507 \\
\hline$<10 \%$ & & & 43 & 5 & \\
\hline $10-30 \%$ & & & 34 & 8 & \\
\hline$>30 \%$ & & & 20 & 2 & \\
\hline Nuclear EGFR & 112 & 5 & & & 0.557 \\
\hline 0 & & & 59 & 8 & \\
\hline+ & & & 22 & 3 & \\
\hline++ & & & 5 & 2 & \\
\hline+++ & & & 11 & 2 & \\
\hline Membrane EGFR & 112 & 5 & & & $0.0420^{\mathrm{a}}$ \\
\hline 0 & & & 86 & 14 & \\
\hline $1+$ & & & 10 & 0 & \\
\hline $2+$ & & & 1 & 0 & \\
\hline $3+$ & & & 0 & 1 & \\
\hline EGFR gene & 110 & 7 & & & 0.255 \\
\hline Not amplified & & & 94 & 14 & \\
\hline Amplified & & & 1 & 1 & \\
\hline HER-2 & 112 & 5 & & & 0.906 \\
\hline 0 & & & 61 & 9 & \\
\hline $1+$ & & & 15 & 2 & \\
\hline $2+$ & & & 8 & 2 & \\
\hline $3+$ & & & 13 & 2 & \\
\hline HER-2 gene & 112 & 5 & & & 0.733 \\
\hline Not amplified & & & 78 & 13 & \\
\hline Amplified & & & 19 & 2 & \\
\hline
\end{tabular}

CCND1, cyclin-D1 gene; EGFR, epidermal growth factor receptor; ER, estrogen receptor; Grade, histological grade; LN mets, lymph node metastasis; LVI, lymphovascular invasion; NA, not available; NPI, Nottingham prognostic index; PR, progesterone receptor. ${ }^{\mathrm{a}} \chi^{2}$-test.

involvement, ER and PR expression, Nottingham prognostic index, lymphovascular invasion, Ki-67, HER-2 and membrane EGFR to examine survival significance. Only strong cyclin-D1 expression paired with ER, PR positivity and low-grade tumors
Table 3 Correlations between nuclear EGFR expression, clinicopathological parameters, EGFR-family proteins and cyclin-D1 in invasive ductal breast cancer

\begin{tabular}{|c|c|c|c|c|c|c|c|}
\hline \multirow[t]{2}{*}{ Parameters } & \multicolumn{4}{|c|}{ No. NA Nuclear Nuclear } & \multicolumn{2}{|c|}{ Nuclear Nuclear } & \multirow{3}{*}{$\begin{array}{c}r \text { Fisher's } \\
\text { exact } \\
\text { test } \\
\text { (P-value) }\end{array}$} \\
\hline & & & $\begin{array}{c}E G F R \\
0\end{array}$ & $\begin{array}{c}E G F R \\
+\end{array}$ & $\begin{array}{c}E G F R \\
++\end{array}$ & $\begin{array}{c}E G F R \\
+++\end{array}$ & \\
\hline Overall & 113 & 4 & 67 & 26 & 7 & 13 & \\
\hline Tumor size & 113 & 4 & & & & & 0.0005 \\
\hline $\mathrm{T} 1(\leqslant 2 \mathrm{~cm})$ & & & 34 & 21 & 2 & 5 & \\
\hline $\mathrm{T} 2(>2-5 \mathrm{~cm})$ & & & 32 & 5 & 3 & 5 & \\
\hline $\mathrm{T} 3(>5 \mathrm{~cm})$ & & & 1 & 0 & 2 & 3 & \\
\hline Grade & 113 & 4 & & & & & 0.9409 \\
\hline I & & & 18 & 8 & 2 & 3 & \\
\hline II & & & 35 & 14 & 3 & 6 & \\
\hline III & & & 14 & 4 & 2 & 4 & \\
\hline LN mets & 113 & 4 & & & & & 0.0288 \\
\hline 0 & & & 37 & 19 & 3 & 2 & \\
\hline $1-3$ & & & 13 & 3 & 1 & 4 & \\
\hline$\geqslant 4$ & & & 17 & 4 & 3 & 7 & \\
\hline$E R$ & 113 & 4 & & & & & 0.4418 \\
\hline - & & & 26 & 10 & 2 & 8 & \\
\hline+ & & & 41 & 16 & 5 & 5 & \\
\hline$P R$ & 113 & 4 & & & & & 0.2088 \\
\hline - & & & 25 & 7 & 5 & 5 & \\
\hline+ & & & 42 & 19 & 2 & 8 & \\
\hline NPI & 112 & 5 & & & & & 0.0011 \\
\hline Good $(<3.4)$ & & & 34 & 17 & 3 & 3 & \\
\hline $\begin{array}{l}\text { Moderate } \\
(3.4-5.4)\end{array}$ & & & 18 & 9 & 0 & 4 & \\
\hline Poor $(>5.4)$ & & & 14 & 0 & 4 & 6 & \\
\hline$L V I$ & 79 & 38 & & & & & 0.9653 \\
\hline - & & & 13 & 5 & 1 & 2 & \\
\hline+ & & & 38 & 12 & 2 & 6 & \\
\hline$K i-67$ & 113 & 4 & & & & & 0.3491 \\
\hline$<10 \%$ & & & 27 & 13 & 2 & 7 & \\
\hline $10-30 \%$ & & & 24 & 11 & 2 & 5 & \\
\hline$>30 \%$ & & & 16 & 2 & 3 & 1 & \\
\hline HER-2 & 112 & 5 & & & & & 0.6488 \\
\hline 0 & & & 41 & 16 & 6 & 7 & \\
\hline $1+$ & & & 11 & 4 & 0 & 2 & \\
\hline $2+$ & & & 6 & 3 & 1 & 0 & \\
\hline $3+$ & & & 9 & 2 & 0 & 4 & \\
\hline HER-2 gene & 113 & 4 & & & & & 0.3128 \\
\hline Not amplified & & & 53 & 23 & 7 & 9 & \\
\hline Amplified & & & 14 & 3 & 0 & 4 & \\
\hline Membrane & 112 & 5 & & & & & 0.9158 \\
\hline EGFR & & & & & & & \\
\hline 0 & & & 57 & 24 & 7 & 12 & \\
\hline $1+$ & & & 8 & 1 & 0 & 1 & \\
\hline $2+$ & & & 1 & 0 & 0 & 0 & \\
\hline $3+$ & & & 1 & 0 & 0 & 0 & \\
\hline EGFR gene & 110 & 7 & & & & & 1 \\
\hline Not amplified & & & 65 & 23 & 7 & 13 & \\
\hline Amplified & & & 2 & 0 & 0 & 0 & \\
\hline Cyclin-D1 & 113 & 4 & & & & & 0.5132 \\
\hline Negative/weak & & & 8 & 2 & 0 & 0 & \\
\hline Moderate & & & 17 & 5 & 3 & 6 & \\
\hline Strong & & & 42 & 19 & 4 & 7 & \\
\hline CCND1 & 112 & 5 & & & & & 0.5569 \\
\hline Not amplified & & & 59 & 22 & 5 & 11 & \\
\hline Amplified & & & 8 & 3 & 2 & 2 & \\
\hline
\end{tabular}

CCND1, cyclin-D1 gene; EGFR, epidermal growth factor receptor; ER, estrogen receptor; Grade, histological grade; LN mets, lymph node metastasis; LVI, lymphovascular invasion; NA, not available; NPI, Nottingham prognostic index; PR, progesterone receptor.

showed statistically significant, better survival as compared with tumors that did not have these histopathological characteristics $\quad(P=0.0422$, 
Table 4 Univariate survival analysis

\begin{tabular}{|c|c|c|c|c|c|}
\hline Overall & No. & Events & $\begin{array}{c}\text { Overall } \\
\text { survival } \\
\text { (mean) } \\
0.81\end{array}$ & $\begin{array}{c}\text { Overall } \\
\text { survival } \\
\text { (s.e.) } \\
0.057\end{array}$ & $\begin{array}{c}\text { Log-rank } \\
\text { test }(\mathrm{P}- \\
\text { value })\end{array}$ \\
\hline Tumor size & 117 & 36 & & & $1.67 \times 10^{-5}$ \\
\hline $\mathrm{T} 1(\leqslant 2 \mathrm{~cm})$ & 63 & 12 & 0.80 & 0.092 & \\
\hline $\mathrm{T} 2(>2-5 \mathrm{~cm})$ & 46 & 18 & 0.79 & 0.076 & \\
\hline Т3 $(>5 \mathrm{~cm})$ & 8 & 6 & 0.52 & 0.378 & \\
\hline Grade & 117 & 36 & & & 0.0030 \\
\hline I & 64 & 12 & 0.83 & 0.042 & \\
\hline II & 21 & 7 & 0.79 & 0.110 & \\
\hline III & 32 & 17 & 0.68 & 0.109 & \\
\hline LN mets & 117 & 36 & & & 0.0024 \\
\hline 0 & 31 & 4 & 0.91 & 0.055 & \\
\hline $1-3$ & 61 & 17 & 0.80 & 0.052 & \\
\hline$\geqslant 4$ & 25 & 15 & 0.64 & 0.138 & \\
\hline$E R$ & 117 & 36 & & & 0.0830 \\
\hline - & 47 & 20 & 0.73 & 0.106 & \\
\hline+ & 70 & 16 & 0.87 & 0.045 & \\
\hline$P R$ & 117 & 36 & & & 0.0007 \\
\hline- & 43 & 21 & 0.71 & 0.089 & \\
\hline+ & 74 & 15 & 0.84 & 0.040 & \\
\hline NPI & 115 & 33 & & & $2.80 \times 10^{-7}$ \\
\hline Good $(<3.4)$ & 59 & 7 & 0.92 & 0.037 & \\
\hline Moderate (3.4-5.4) & 31 & 10 & 0.81 & 0.085 & \\
\hline Poor $(>5.4)$ & 25 & 16 & 0.64 & 0.153 & \\
\hline$L V I$ & 83 & 25 & & & 0.5058 \\
\hline - & 22 & 5 & 0.84 & 0.092 & \\
\hline+ & 61 & 20 & 0.79 & 0.057 & \\
\hline $\mathrm{Ki}-67$ & 113 & 35 & & & 0.4845 \\
\hline$<10 \%$ & 49 & 13 & 0.79 & 0.056 & \\
\hline $10-30 \%$ & 42 & 12 & 0.83 & 0.069 & \\
\hline$>30 \%$ & 22 & 10 & 0.69 & 0.121 & \\
\hline HER-2 & 116 & 36 & & & 0.2667 \\
\hline 0 & 71 & 21 & 0.79 & 0.080 & \\
\hline $1+$ & 18 & 4 & 0.84 & 0.100 & \\
\hline $2+$ & 11 & 3 & 0.80 & 0.152 & \\
\hline $3+$ & 16 & 8 & 0.69 & 0.167 & \\
\hline HER-2 gene & 116 & 36 & & & 0.0522 \\
\hline Not amplified & 93 & 26 & 0.81 & 0.066 & \\
\hline Amplified & 21 & 10 & 0.73 & 0.129 & \\
\hline Cyclin-D1 & 113 & 35 & & & 0.1538 \\
\hline Negative/weak & 10 & 5 & 0.66 & 0.226 & \\
\hline Moderate & 31 & 12 & 0.73 & 0.090 & \\
\hline Strong & 72 & 18 & 0.82 & 0.045 & \\
\hline CCND1 & 112 & 35 & & & 0.3986 \\
\hline Not amplified & 97 & 29 & 0.80 & 0.065 & \\
\hline Amplified & 15 & 6 & 0.74 & 0.151 & \\
\hline Nuclear EGFR & 113 & 35 & & & 0.0003 \\
\hline 0 & 67 & 20 & 0.83 & 0.053 & \\
\hline+ & 26 & 3 & 0.70 & 0.051 & \\
\hline++ & 7 & 3 & 0.57 & 0.211 & \\
\hline+++ & 13 & 9 & 0.58 & 0.249 & \\
\hline $\begin{array}{l}\text { Cyclin-D1 pre- } \\
\text { menopause }\end{array}$ & 54 & 11 & & & 0.0033 \\
\hline Negative/weak & 3 & 2 & 0.44 & 0.680 & \\
\hline Moderate & 15 & 4 & 0.68 & 0.107 & \\
\hline Strong & 36 & 5 & 0.76 & 0.047 & \\
\hline $\begin{array}{l}\text { Cyclin-D1 post- } \\
\text { menopause }\end{array}$ & 59 & 24 & & & 0.5365 \\
\hline Negative/weak & 7 & 3 & 0.67 & 0.262 & \\
\hline Moderate & 16 & 8 & 0.69 & 0.167 & \\
\hline Strong & 36 & 13 & 0.78 & 0.086 & \\
\hline $\begin{array}{l}\text { Nuclear EGFR } \\
\text { premenopause }\end{array}$ & 54 & 11 & & & $3.58 \times 10^{-6}$ \\
\hline 0 & 30 & 4 & 0.91 & 0.057 & \\
\hline+ & 16 & 1 & 0.50 & 0.000 & \\
\hline++ & 2 & 1 & 0.50 & 0.00 & \\
\hline+++ & 6 & 5 & 0.44 & 0.55 & \\
\hline
\end{tabular}

Table 4 Continued

\begin{tabular}{|c|c|c|c|c|c|}
\hline Overall & No. & Events & $\begin{array}{c}\text { Overall } \\
\text { survival } \\
\text { (mean) } \\
0.81\end{array}$ & $\begin{array}{c}\text { Overall } \\
\text { survival } \\
\text { (s.e.) } \\
0.057\end{array}$ & $\begin{array}{c}\text { Log-rank } \\
\text { test }(\mathrm{P}- \\
\text { value) }\end{array}$ \\
\hline $\begin{array}{l}\text { Nuclear EGFR } \\
\text { postmenopause }\end{array}$ & 59 & 24 & & & 0.3377 \\
\hline 0 & 37 & 16 & 0.75 & 0.094 & \\
\hline+ & 10 & 2 & 0.83 & 0.141 & \\
\hline++ & 5 & 2 & 0.67 & 0.318 & \\
\hline+++ & 7 & 4 & 0.60 & 0.319 & \\
\hline
\end{tabular}

CCND1, cyclin-D1 gene; EGFR, epidermal growth factor receptor; ER, estrogen receptor; Grade, histological grade; LN mets, lymph node metastasis; LVI, lymphovascular invasion; NA, not available; NPI, Nottingham prognostic index; PR, progesterone receptor.

$0.0145,0.0192$, respectively; data shown in the Supplementary information). In our study group we did not find statistically significant correlation in the context of overall survival when cyclin-D1 or CCND1 gene status were pared with HER-2 and membrane EGFR protein overexpression or gene amplification. However, CCND1 amplification failed to show any correlation with overall survival (Table 4). Tumors with high nuclear EGFR staining in the same cohort showed worse overall patient survival compared with those without detectable nuclear EGFR $(P=0.0003)$. Nuclear EGFR was shown to be a significantly worse overall survival factor also in premenopausal women $(P<0.00001)$ (Table 4). Tumors with moderate/strong expression of cyclinD1 and nuclear EGFR had significantly shorter cumulative survival compared with tumors with moderate/strong cyclin-D1 expression and negative/ weak nuclear EGFR expression $(P=0.00078)$ (Figure 2). Multivariate analysis revealed only tumor size, histological grade, Nottingham prognostic index and nuclear EGFR as independent prognostic factors for overall survival (Table 5). In our cohort, nuclear EGFR +++ patients had mortality risk 3.4 times greater than nuclear EGFR-negative patients (hazard ratio $=3.402 ; P=0.0026$ ).

\section{Discussion}

Proteins from the EGFR family and cyclin-D1 are often deregulated in human breast cancer and frequently correlations between expression of these proteins and clinical outcome have been inconsistent, ${ }^{4}$ mainly due to differing laboratory methodologies, non-uniform thresholds for positivity and different patient groups. Thus, it is of great importance to further evaluate the complex associations and prognostic implications of EGFR-family protein members and cyclin-D1. There is emerging evidence that EGFR-family proteins function not only as signaling initiators on the cell membrane, but also undergo nuclear translocation and subsequently 
a
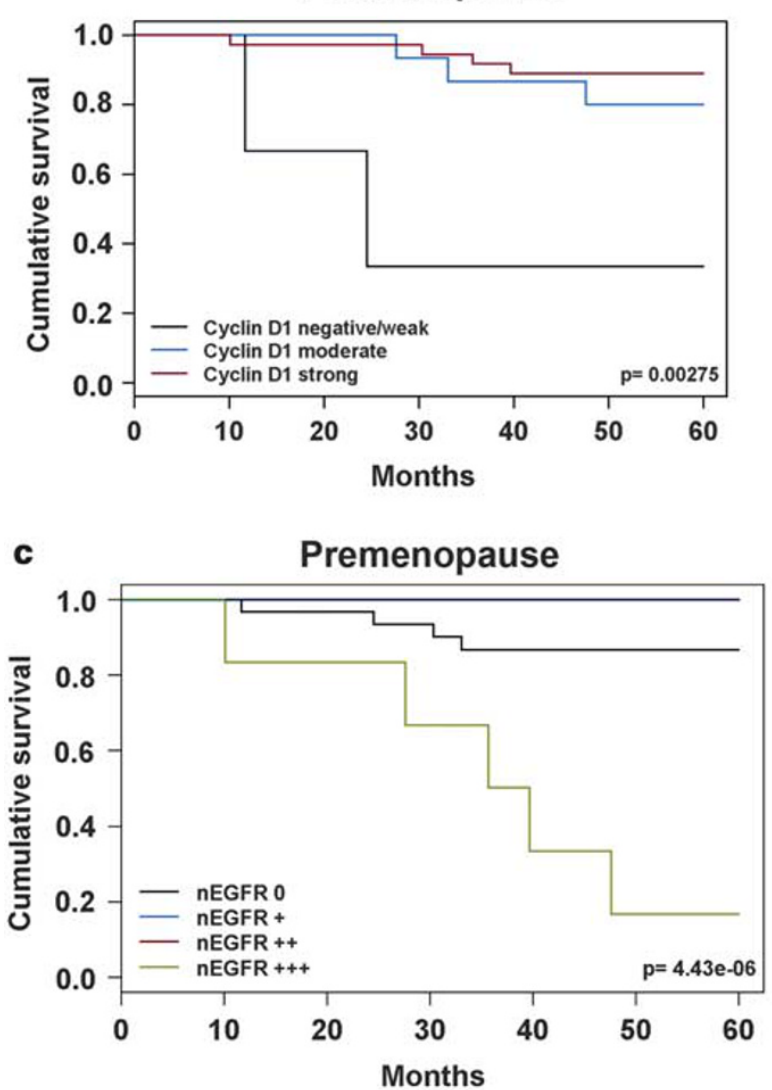

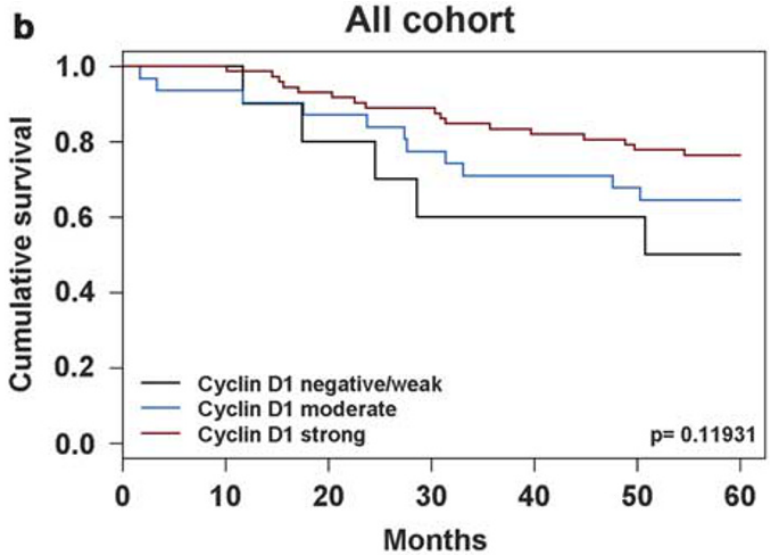

d $\quad$ All cohort

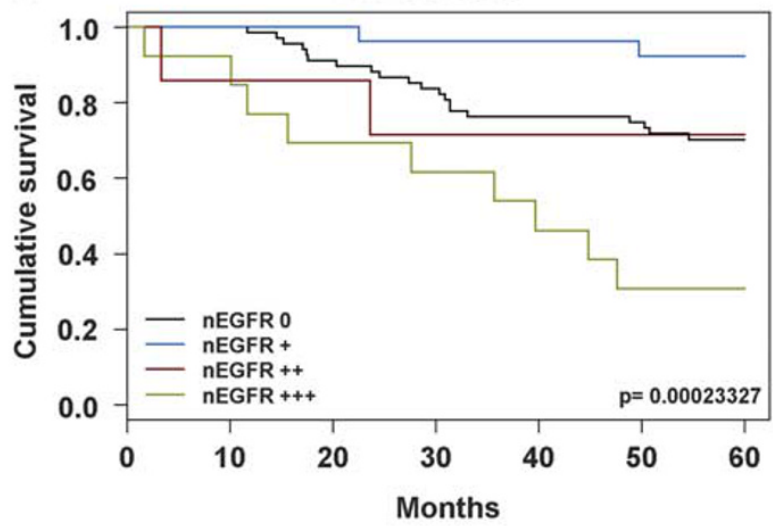

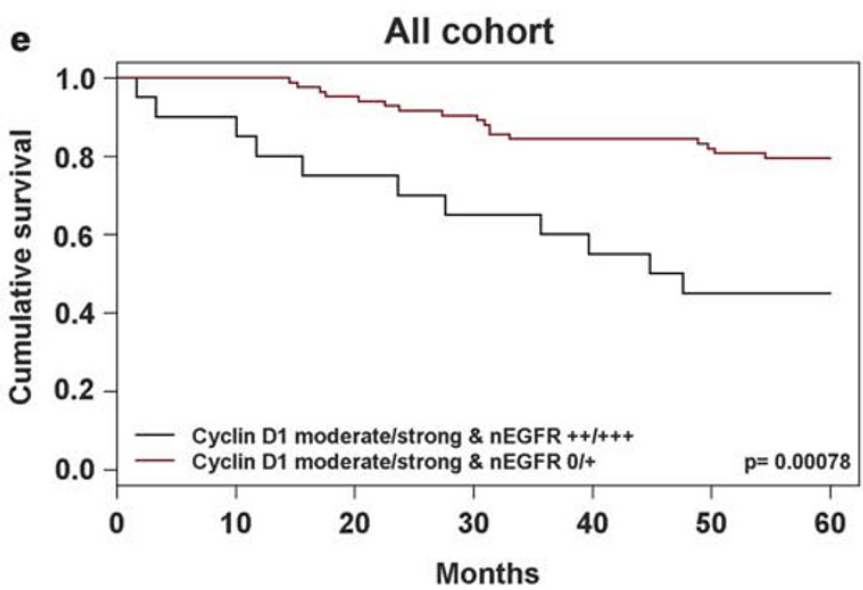

Figure 2 Univariate analysis of the prognostic impact of cyclin-D1 overexpression and nuclear EGFR staining, individually and in combination, on overall survival. Cyclin-D1 expression and overall survival in the premenopausal group of patients (a) and in the whole cohort of patients (b). Nuclear EGFR staining and overall survival in the premenopausal group of patients (lines for + nuclear EGFR and ++ nuclear EGFR are overlapping, producing one purple line) (c) and in the whole cohort of patients (d). Overall survival of patents with cyclin-D1 moderate/strong expression and 0/ + nuclear EGFR staining, with survival of patients with cyclin-D1 moderate/strong and $++I+++$ nuclear EGFR $(\mathbf{e})$.

regulate gene expression and potentially mediate other cellular processes. ${ }^{26,27}$

The recent study by Lo et $a l^{19}$ is the first, and to our knowledge only, study to describe the prognostic value of nuclear EGFR in breast cancer patients, demonstrating that tumors with high nuclear EGFR staining had increased proliferative potential with worse overall survival. They showed positive correlation of nuclear EGFR with increased levels of cyclin-D1 and Ki-67; however, our data did not confirm such a correlation..$^{19}$ It is difficult to discuss and compare our findings regarding the correlation 
Table 5 Cox proportional hazards analysis for predictors of overall survival

\begin{tabular}{lccc}
\hline Variables & $\begin{array}{c}\text { Hazards } \\
\text { ratio }\end{array}$ & $\begin{array}{c}95 \% \\
\text { confidence } \\
\text { interval }\end{array}$ & P-value \\
\hline Tumor size $>2 \mathrm{~cm}^{\mathrm{a}}$ & 3.09 & $2.054-4.654$ & 0.0058 \\
Grade-III $^{\mathrm{b}}$ & 6.90 & $3.635-13.103$ & 0.0026 \\
Nottingham prognostic index $^{\mathrm{c}}$ & 2.96 & $1.810-4.853$ & 0.0280 \\
Nuclear EGFR $+++^{\mathrm{d}}$ & 3.40 & $2.264-5.109$ & 0.0026
\end{tabular}

EGFR, epidermal growth factor receptor.

${ }^{\mathrm{a}}$ Compared with tumor size $\leqslant 2 \mathrm{~cm}$.

${ }^{\mathrm{b}}$ Compared with grade-I.

${ }^{\mathrm{C}}$ Fitted as linear term, that is, increase in risk for change in grade of one unit.

${ }^{\mathrm{d}}$ Compared with nuclear EGFR-negative tumors.

of cyclin-D1 and Ki-67 to nuclear EGFR with those of Lo et al since they have not clearly stated cyclinD1 and Ki-67 immunohistochemistry scoring and thresholds.

Cyclin-D1 is one of the frequently overexpressed proteins and one of the commonly amplified genes in breast cancer. In our study, $64 \%$ of the cases showed strong cyclin-D1 expression, whereas only $9 \%$ showed very low or no expression of this cellcycle regulator. On the other hand, the frequency of CCND1 amplification, as detected by FISH in a same cohort of patients, was $13 \%$, which is similar to previous reports. ${ }^{13,24}$ Discrepancy between cyclinD1 protein expression and gene amplification has previously been shown and it suggests that mechanisms other than gene amplification are responsible for the altered expression observed in some cases. ${ }^{28}$ Strong cyclin-D1 expression in the majority of breast cancers, as detected in this study, is not surprising given that this protein plays an important role in the progression from $G_{1}$ to $S$ phase and can be upregulated through several different pathways, including EGFR-family protein, ER, c-myc and fibroblast growth factor receptor pathways. ${ }^{13,19,24}$ Our study showed a significant correlation between cyclin-D1 protein expression and CCND1 amplification, and all cases having amplification showed either moderate or strong cyclin-D1 expression.

It has been reported that ER expression is associated with cyclin-D1 overexpression. ${ }^{29,30}$ CCND1 is a transcriptional target of ER and this process seems to be mediated by recruitment of specific ER coactivators and activation of IKK $\alpha$ and MAPK pathways. ${ }^{31,32}$ On the other hand cyclin-D1 also binds to ER and activates it in a hormoneindependent manner. ${ }^{24,33}$ In our study we have confirmed a significant association between cyclinD1 overexpression and ER positivity, and have also found an inverse correlation with the Nottingham prognostic index and membrane EGFR. However, we did not find significant association between CCND1 amplification and ER positivity, but CCND1 amplification showed inverse correlation with membrane EGFR. Although, some studies have reported that cyclin-D1 overexpression indicates poor prognosis in breast cancer, ${ }^{16,34,35}$ the majority failed to confirm a relationship between cyclin-D1 overexpression and shortened survival. ${ }^{13,28,36}$ In the present study, cyclin-D1 overexpression significantly correlated with better overall survival in the premenopausal group of women, but there was no significant correlation in the post-menopausal group or the whole patient cohort, although a trend toward better survival has been noticed (Table 4). When we paired established histopathological parameters like ER and PR expression, and low histological differentiation with strong cyclin-D1 expression, those patients showed better survival suggesting that high cyclin-D1 expression is a good prognostic indicator.

These results seem contradictory considering the well known function of cyclin-D1 in cell-cycle regulation, activating the cascade of events that lead to DNA replication and cell division. However, some studies suggest that cyclin-D1 may have antitumor effects. Turner et $a l^{37}$ suggested that high expression of cyclin-D1 can make the tumor more sensitive to radiation, while Lin et $a l^{38}$ suggested that cyclin-D1 may lead to induction of apoptosis, cellular senescence and cellular growth inhibition. In our study we did not find a correlation between cyclin-D1 expression and cell proliferation activity mirrored by Ki-67, which supports the interpretation that cyclin-D1 might demonstrate other functions that are not related to cell-cycle progression and tumor aggressiveness. However, we did not find a correlation between CCND1 and overall survival. The prognostic impact of CCND1 amplification is indirectly in agreement with previous studies showing that CCND1 amplification is not associated with poor prognosis. ${ }^{13,39}$ Although experimental data indicate that cyclin-D1 is a major downstream target of EGFR-family-dependent signaling, we did not observe a statistically significant association between HER-2 and cyclin-D1, but did observe an inverse correlation with membrane EGFR and cyclin-D1 expression.

In our study $2 \%$ of tumors showed membrane EGFR overexpression, which correlated well with gene amplification $(2 \%)$ and these results are in agreement with the study by Al Kuraya et $a .^{39}$ Studies that have reported higher percentage of membrane EGFR expression besides different methodologies used, also used not so stringent criteria in defining membrane EGFR overexpression and interpreted overexpresison as more than $1 \%$ of positive cells with any membrane staining. ${ }^{3,7}$ In our study, $9 \%$ tumors with $1+$ staining were interpreted as negative for membrane EGFR overexpression, which is the major contributing factor for such low prevalence of membrane EGFR overexpression. However, some report that even low-level expression of membrane EGFR may still play an important role in the biology of a tumor, especially in the context of expression and overexpression of other 
EGFR-family members. ${ }^{40}$ The discrepancy between the number of cases expressing membrane EGFR (2\%) and nuclear EGFR (40\%) suggests that in tumors with low level of membrane EGFR expression, the mechanisms involved in EGFR trafficking and receptor translocation might be more important in signal transmission, leading to even more aggressive tumor behavior. This finding also further emphasizes the relevance of the new direct EGFRsignaling pathway, which involves direct shuttling of activated EGFR into the nucleus, especially in the light of often lacking correlation of membrane EGFR expression with prognosis.

In our study we did not find a correlation between membrane EGFR and HER-2, and clinical significance of membrane EGFR overexpression and EGFR amplification could not be independently evaluated due to small number of informative cases. Besides well established traditional EGFR-signaling pathway, emerging evidence suggests the existence of a direct mode of EGFR signaling where EGFR is transported from the cell surface to the nucleus. The nuclear counterpart of EGFR appears to be the full-length protein and likely in the phosphorylated form as shown by a number of studies, ${ }^{11,41}$ but it is not yet clear by which cellular mechanism or why cell-surface EGFR gains entry. ${ }^{11,41,42}$ Nuclear translocation is not a unique event with EGFR, but rather a universal phenomenon occurring for many other cell-surface receptors. ${ }^{11}$ EGFR is translocated to the nucleus upon ligand stimulation, but in addition nuclear translocation of EGFR can be initiated by irradiation, heat shock, $\mathrm{H}_{2} \mathrm{O}_{2}$ and cisplatin. ${ }^{12,41,43}$ In our study we have detected nuclear EGFR staining in $40 \%$ of breast cancer samples and $12 \%$ samples had high-level nuclear EGFR staining $(+++)$, which is in accordance with previous report. ${ }^{19} \mathrm{We}$ found only two cases in which EGFR was amplified, while $40 \%$ of the tumors studied were immunohistochemically positive for nuclear EGFR, suggesting that gene amplification is not a significant event that would lead to higher nuclear translocation of the EGFR cell-surface receptor. The question is what actually happened in tumors that were membrane EGFR-negative and showed moderate/strong nuclear EGFR staining? Maybe those tumors had increased transcription of the EGFR gene, but subsequent posttranscriptional modifications occurred, which diverted EGFR from its traditional path onto the cell surface, directly into the nucleus. This might indirectly indicate that trafficking-dependent alterations in cell-surface receptor are crucial regulatory mechanisms in EGFR signaling.

The results of Kaplan-Meier survival analysis and log-rank test revealed a significant inverse correlation between high nuclear EGFR and overall survival in the whole cohort, as reported earlier, ${ }^{19}$ and in the premenopausal group of patients. By multivariate analysis we found nuclear EGFR to be an independent prognostic factor for patient overall survival. These observations come into light of the report showing that anti-EGFR antibody (C255/ Cetuximab) blocks radiation-induced nuclear EGFR transport and its interaction with DNA-dependent protein kinase, an enzyme involved in DNA repair, and thus may lead to radiosensitization. ${ }^{41,44}$ Interestingly, besides correlation between nuclear EGFR and tumor size, lymph nodes and Nottingham prognostic index, we found a correlation between nuclear EGFR and ER in the premenopausal group of women. Patients with tumors expressing a moderate-to-strong level of cyclin-D1 protein and moderate-to-strong nuclear EGFR staining had significantly worse survival compared with patients whose tumors had moderate/strong cyclin-D1 expression and negative to low nuclear EGFR. These data are in agreement with earlier studies that showed association of nuclear EGFR with the promoter region of cyclin-D1, and direct activation of transcription of this cell-cycle protein. ${ }^{12}$ It has been shown that nuclear EGFR functions as a transcriptional cofactor that interacts with and activates not only the CCND1 but also the iNOS gene. ${ }^{11,42}$ The nuclear EGFR complex specifically and strongly recognizes an AT-rich response sequence, designated ATRS, present in the promoters of the CCND1 and iNOS gene. ${ }^{11,42}$ Also, our data indicate that in the context of high nuclear EGFR staining, increased expression of cyclin-D1 actually contributes to poor survival rate in invasive ductal breast cancer. We postulate that in these cases nuclear EGFR could lead to overexpression of cyclin-D1 through transcriptional activation of $C C N D 1$, which consequently leads to more aggressive tumor behavior and subsequent decreased patient survival. Also, nuclear localization and action of EGFR constitute a potential mechanism of resistance to EGFR-targeted therapies, since nuclear EGFR directly activates transcription bypassing the protein phosphorylation cascade; thus, EGFR-rich tumors may not respond to EGFR inhibitors blocking only receptor-mediated signaling. ${ }^{45}$

In conclusion, this study confirms that strong cyclin-D1 expression represents a good prognostic marker. However, when paired with strong nuclear EGFR, cyclin-D1-overexpressing tumors confer a more aggressive clinical behavior leading to worse patient overall survival. Our data show strong statistical correlation between high nuclear EGFR staining and worse overall survival in breast cancer patients.

\section{Acknowledgements}

We thank our technicians Tanja Kovačević, Ozren Štanfel and Ankica Brajenić for technical assistance. This work was supported by the Ministry of Science, Education and Sports of the Republic of Croatia (grant numbers 062-0620095-0077 and 062-00000003550). 


\section{Disclosure/conflict of interest}

The authors declare no conflict of interest.

\section{References}

1 WHO. Cancer database fact sheet N 297 February 2006. Available from URL:http://who.int/mediacentre/fact sheets/fs297/en/accessed 19.05.2009.

2 Ferlay J, Bray F, Pisani P, et al. GLOBOCAN 2002: Cancer Incidence, Mortality and Prevalence Worldwide, IARC CancerBase No.5.Version 2.0. IARCPress: Lyon, France, 2004.

3 Milanezi F, Carvalho S, Schmitt FC. EGFR/HER2 in breast cancer: a biological approach for molecular diagnosis and therapy. Expert Rev Mol Diagn 2008;8:417-434.

4 Cho EY, Choi YL, Han JJ, et al. Expression and amplification of Her2, EGFR and cyclin D1 in breast cancer: immunohistochemistry and chromogenic in situ hybridization. Pathol Int 2008;58:17-25.

5 Järvinen TA, Liu ET. HER-2/neu and topoisomerase IIalpha-simultaneous drug targets in cancer. Comb Chem High Throughput Screen 2003;6:455-470.

6 Ross JS, Fletcher JA, Bloom KJ, et al. Targeted therapy in breast cancer: the HER-2/neu gene and protein. Mol Cell Proteomics 2004;3:379-398.

7 Bhargava R, Gerald WL, Li AR, et al. EGFR gene amplification in breast cancer: correlation with epidermal growth factor receptor mRNA and protein expression and HER-2 status and absence of EGFRactivating mutations. Mod Pathol 2005;18:1027-1033.

8 Chan SK, Hill ME, Gullick WJ. The role of the epidermal growth factor receptor in breast cancer. J Mammary Gland Biol Neoplasia 2006;11:3-11.

9 Suo Z, Risberg B, Kalsson MG, et al. EGFR family expression in breast carcinomas. c-erbB-2 and c-erbB-4 receptors have different effects on survival. J Pathol 2002;196:17-25.

10 Buchholz TA, Tu X, Ang Kk, et al. Epidermal growth factor receptor expression correlates with poor survival in patients who have breast carcinoma treated with doxorubicin-based neoadjuvant chemotherapy. Cancer 2005;104:676-681.

11 Lo HW, Hung MC. Nuclear EGFR signalling network in cancers: linking EGFR pathway to cell cycle progression, nitric oxide pathway and patient survival. Br J Cancer 2006;94:184-188.

12 Lin SY, Makino K, Xia W, et al. Nuclear localization of EGF receptor and its potential new role as a transcription factor. Nat Cell Biol 2001;3:802-808.

13 Reis-Filho JS, Savage K, Lambros MB, et al. Cyclin D1 protein overexpression and CCND1 amplification in breast carcinomas: an immunohistochemical and chromogenic in situ hybridisation analysis. Mod Pathol 2006;19:999-1009.

14 Jirström K, Stendahl M, Rydén L, et al. Adverse effect of adjuvant tamoxifen in premenopausal breast cancer with cyclin D1 gene amplification. Cancer Res 2005;65:8009-8016.

15 Stendahl M, Kronblad A, Rydén L, et al. Cyclin D1 overexpression is a negative predictive factor for tamoxifen response in postmenopausal breast cancer patients. Br J Cancer 2004;90:1942-1948.

16 Ahnström M, Nordenskjöld B, Rutqvist LE, et al. Role of cyclin D1 in ErbB2-positive breast cancer and tamoxifen resistance. Breast Cancer Res Treat 2005;91:145-151.

17 Lee A, Park WC, Yim HW, et al. Expression of c-erbB2, cyclin D1 and estrogen receptor and their clinical implications in the invasive ductal carcinoma of the breast. Jpn J Clin Oncol 2007;37:708-714.

18 Cho EY, Han JJ, Choi YL, et al. Comparison of Her-2, EGFR and cyclin D1 in primary breast cancer and paired metastatic lymph nodes: an immunohistochemical and chromogenic in situ hybridization study. J Korean Med Sci 2008;23:1053-1061.

19 Lo HW, Xia W, Wei Y, et al. Novel prognostic value of nuclear epidermal growth factor receptor in breast cancer. Cancer Res 2005;65:338-348.

20 Ellis IO, Galea M, Broughton N, et al. Pathological prognostic factors in breast cancer. II. Histological type. Relationship with survival in a large study with long-term follow-up. Histopathology 1992;20: 479-489.

21 Singletary SE, Connolly JL. Breast cancer staging: working with the sixth edition of the AJCC Cancer Staging Manual. CA Cancer J Clin 2006;56: 37-47.

22 Blamey RW, Ellis IO, Pinder SE, et al. Survival of invasive breast cancer according to the Nottingham Prognostic Index in cases diagnosed in 1990-1999. Eur J Cancer 2007;43:1548-1555.

23 Wolff AC, Hammond ME, Schwartz JN, et al. American Society of Clinical Oncology; College of American Pathologists. American Society of Clinical Oncology/ College of American Pathologists guideline recommendations for human epidermal growth factor receptor 2 testing in breast cancer. J Clin Oncol 2007;25: 118-145.

24 Elsheikh S, Green AR, Aleskandarany MA, et al. CCND1 amplification and cyclin D1 expression in breast cancer and their relation with proteomic subgroups and patient outcome. Breast Cancer Res Treat 2008;109:325-335.

25 Liu K, Muse SV. PowerMarker: integrated analysis environment for genetic marker data. Bioinformatics 2005;21:2128-2129.

26 Carpenter G. Nuclear localization and possible functions of receptor tyrosine kinases. Curr Opin Cell Biol 2003;15:143-148.

27 Clevenger CV. Nuclear localization and function of polypeptide ligands and their receptors: a new paradigm for hormone specificity within the mammary gland? Breast Cancer Res 2003;5:181-187.

28 Hwang TS, Han HS, Hong YC, et al. Prognostic value of combined analysis of cyclin D1 and estrogen receptor status in breast cancer patients. Pathol Int 2003;53: 74-80.

29 Doisneau-Sixou SF, Sergio CM, Carroll JS, et al. Estrogen and antiestrogen regulation of cell cycle progression in breast cancer cells. Endocr Relat Cancer 2003;10:179-186.

30 Lebeau A, Unholzer A, Amann G, et al. EGFR, HER-2/ neu, cyclin D1, p21 and p53 in correlation to cell proliferation and steroid hormone receptor status in ductal carcinoma in situ of the breast. Breast Cancer Res Treat 2003;79:187-198.

31 Cicatiello L, Addeo R, Sasso A, et al. Estrogens and progesterone promote persistent CCND1 gene activation during G1 by inducing transcriptional derepression via c-Jun/c-Fos/estrogen receptor (progesterone receptor) complex assembly to a distal regulatory 
element and recruitment of cyclin D1 to its own gene promoter. Mol Cell Biol 2004;24:7260-7274.

32 Park KJ, Krishnan V, O’Malley BW, et al. Formation of an IKKalpha-dependent transcription complex is required for estrogen receptor-mediated gene activation. Mol Cell 2005;18:71-82.

33 Zwijsen RM, Buckle RS, Hijmans EM, et al. Ligandindependent recruitment of steroid receptor coactivators to estrogen receptor by cyclin D1. Genes Dev 1998;12:3488-3498.

34 Kenny FS, Hui R, Musgrove EA, et al. Overexpression of cyclin D1 messenger RNA predicts for poor prognosis in estrogen receptor-positive breast cancer. Clin Cancer Res 1999;5:2069-2076.

35 Guo LL, Gao P, Wu YG, et al. Alteration of cyclin D1 in Chinese patients with breast carcinoma and its correlation with Ki-67, pRb, and p53. Arch Med Res 2007;38:846-852.

36 Park K, Han S, Kim HY, et al. Cytologic evaluation of cyclin D1 expression in primary breast carcinoma. Cancer 2001;93:211-215.

37 Turner BC, Gumbs AA, Carter D, et al. Cyclin D1 expression and early breast cancer recurrence following lumpectomy and radiation. Int J Radiat Oncol Biol Phys 2000;47:1169-1176.

38 Lin HM, Lee YJ, Li G, et al. Bcl-2 induces cyclin D1 promoter activity in human breast epithelial cells independent of cell anchorage. Cell Death Differ 2001;8:44-50.
39 Al-Kuraya K, Schraml P, Torhorst J, et al. Prognostic relevance of gene amplifications and coamplifications in breast cancer. Cancer Res 2004;64:8534-8540.

40 DiGiovanna MP, Stern DF, Edgerton SM, et al. Relationship of epidermal growth factor receptor expression to ErbB-2 signaling activity and prognosis in breast cancer patients. J Clin Oncol 2005;23: 1152-1160.

41 Dittmann K, Mayer C, Fehrenbacher B, et al. Radiationinduced epidermal growth factor receptor nuclear import is linked to activation of DNA-dependent protein kinase. J Biol Chem 2005;280:31182-31189.

42 Lo HW, Hsu SC, Hung MC. EGFR signalling pathway in breast cancers: from traditional signal transduction to direct nuclear translocalization. Breast Cancer Res Treat 2006;95:211-218.

43 Cao H, Lei ZM, Bian L, et al. Functional nuclear epidermal growth factor receptors in human choriocarcinoma JEG-3 cells and normal human placenta. Endocrinology 1995;136:3163-3172.

44 Dittmann K, Mayer C, Rodemann HP. Inhibition of radiation-induced EGFR nuclear import by C225 (cetuximab) suppresses DNA-PK activity. Radiother Oncol 2005;76:157-161.

45 Psyrri A, Yu Z, Weinberger PM, et al. Quantitative determination of nuclear and cytoplasmic epidermal growth factor receptor expression in oropharyngeal squamous cell cancer by using automated quantitative analysis. Clin Cancer Res 2005;11:5856-5862.

Supplementary Information accompanies the paper on Modern Pathology website (http://www.nature.com/ modpathol) 